\title{
A short-turning policy for the management of demand disruptions in rapid transit systems
}

\author{
David Canca • Eva Barrena • Gilbert Laporte • \\ Francisco A. Ortega
}

\begin{abstract}
Rapid transit systems timetables are commonly designed to accommodate passenger demand in sections with the highest passenger load. However, disruptions frequently arise due to an increase in the demand, infrastructure incidences or as a consequence of fleet size reductions. All these circumstances give rise to unsupplied demand at certain stations, which generates passenger overloads in the available vehicles. The design of strategies that guarantee reasonable user waiting time with small increases of operation costs is now an important research topic. This paper proposes a tactical approach to determine optimal policies for dealing with such situations. Concretely, a short-turning strategy is analysed, where some vehicles perform short cycles in order to increase the frequency among certain stations of the lines and to equilibrate the train occupancy level. Turn-back points should be located and service offset should be determined with the objective of diminishing the passenger waiting time while preserving certain level of quality of service. Computational results and analysis for a real case study are provided.
\end{abstract}

Keywords Railways · Timetabling · Short-turning · Disruptions

D. Canca

Industrial Engineering and Management Science I, School of Engineering, University of Seville, Av. de los Descubrimientos s/n, 41092 Seville, Spain

e-mail:dco@us.es

E. Barrena $\cdot$ G. Laporte

Interuniversity Research Center on Network Enterprise, Logistics and Transportation (CIRRELT) and HEC Montréal, 3000 chemin de la Côte-Sainte-Catherine, Montréal , QC H3T 2A7, Canada e-mail: eva.barrena@cirrelt.ca

G. Laporte

e-mail: gilbert.laporte@ cirrelt.ca

F. A. Ortega $(\otimes)$

Department of Applied Mathematics I, School of Architecture, University of Seville, Av. Reina Mercedes

2, 41012 Seville, Spain

e-mail: riejos@us.es 


\section{Introduction}

The aim of the global transit planning process is the determination of a set of lines and the design of services to satisfy a certain transportation demand. The transport offer materializes into a set of timetables to which vehicles and drivers are assigned, subject to a wide set of complex constraints related to infrastructure and vehicle characteristics. As was pointed out by Ceder and Wilson (1986), this process can be decomposed into a sequence of five successive stages, namely: route (network) design, line planning, scheduling and timetabling, rollingstock and crew scheduling, and rostering (see also Bussieck et al. 1997; Bussieck 1998; Desaulniers and Hickman 2007). Due to the exceptional complexity of the whole process, it is impractical to simultaneously solve all these problems (see Magnanti and Wong 1984; Quak 2003). For this reason, a hierarchical approach that solves a sequence of subproblems has been traditionally applied to deal with the full planning process, despite of the obvious fact that optimality cannot be guaranteed, as stated by Guihaire and Hao (2008). Recently, there have been some attempts to study more than one stage simultaneously. Thus Liebchen and Möhring (2007) deal with periodic vehicle scheduling and line planning, Michaelis and Schöbel (2009) integrate routing, line design and timetabling, Vera (2010) manages in the same model crew scheduling and rostering, and Cadarso and Marín (2012) jointly analyze timetable planning and rolling stock.

Disruptions appear to be frequently due to circumstances such as an increase in the demand, infrastructure malfunctioning or the need to reduce the fleet size. These situations give rise to unsupplied demand at certain stations and consequently to passenger overloads in the available vehicles, resulting in an increase in passenger average waiting time or a diminution of their comfort level. In fact, these are fundamental objectives in the robustness analysis of rapid transit systems (RTS) (see Fischetti et al. 2009; Kroon et al. 2007; Liebchen and Stiller 2009; Mesa and Ortega 2013). Therefore, designing strategies that guarantee admissible user waiting times, while maintaining reasonable operation costs, has been one of the major challenges faced by the public transportation agencies.

Short-turning is a convenient tactical policy when high demand zones need to be serviced whereas other zones present a low demand (e.g., lines connecting distant residential areas with city centers or lines connecting two main intermodal transportation hubs). Under this policy, some vehicles can perform short cycles in order to increase the frequency in specific zones of the line, thus diminishing the average waiting time in these zones (Furth 1987). Short-turning is typically designed in advance, with the additional objective of equilibrating vehicle occupancy levels over all the segments of the line. In this paper, short-turning will be applied not as a means of dealing with usual demand conditions, but as a method to manage passenger overloads after an episode of demand increase.

The literature in which short-turning is analyzed in a bus context is extensive (see Ceder 1989; Chen and Niu 2009; Cortés et al. 2011; Delle Site and Filippi 1998; Tirachini and Cortés 2007; Tirachini et al. 2011). From a railway perspective there have been fewer contributions. Coor (1997) provides a review of the utilization of this tactical decision at railway networks. In general, the constraints imposed by the railway infrastructure preclude a generalized application of the results obtained for the bus case to RTS. This includes, for example, the analysis of frequency determination, whose solution requires a further procedure in order to be implemented in a real railway scenario. Mesa et al. (2009) formulate a multicriteria optimization model which integrates two control strategies (short-turning and expressing, or dynamic stop skipping) in order to adapt the frequency distribution of trains along a corridor to the existing trip demand. Hadas and Ceder (2010) develop a dynamic programming model for minimizing the total average travel time required in the operations between three connected 
lines, where several parameters produce different real scenarios of synchronization (or not) that are analyzed by using simulation. Canca et al. (2012) deal with the problem of inserting special services (shuttles) in a two-direction transit corridor, by means of an optimization model that combines both control strategies, short-turning and deadheading. In general, due to their analytical complexity, approaches to short-turning methods in the context of railways are based on concrete illustrations and there are no general models that can be applied without modifications to every situation.

Others strategies for alleviating the situation of overloaded stops have been proposed. For instance, skipping stops (deadheading) at those stations with less demand, holding vehicles at a station, adding vehicles held in reserve or splitting trains. Eberlein et al. (1998) formulate the stop-skipping problem as an integer quadratic programming model with non-linear constraints. Eberlein et al. (1999) investigate the deadheading problem as a control strategy in a real-time scenario. Fu et al. (2003) introduce a new transit operating strategy, where line runs operate in pairs: the first vehicle provides a local service for all stops, whereas for the second train is allowed to skip some stations; the associated approach is modeled as a non-linear integer programming problem. Furth (1987) provides a methodology for computing the requirements of bus fleet and determining a deadheading schedule in order to balance the passenger flows. Wilson et al. (1992) develop a model to simulate the MBTA (Massachusetts Bay Transportation Authority) Green Line, for evaluating the effectiveness of different strategies, such as deadheading, expressing, and short-turning, by minimizing passenger traveling time taking into consideration both ride time and waiting time with equal weights. Next, Soeldner (1993) evaluates short-turning decisions on the MBTA Green Line.

The insertion of vehicles into existing timetables has been analyzed by several authors. Thus, Zhou and Zhong (2005) studied the coexistence of high speed and regular trains in a railway corridor giving priority to the high speed trains timetables. Canca (2009) studied the incorporation of new services in a network without alteration of the existing timetables (see also Flier et al. 2009 and Cacchiani et al. 2010b).

Since bus travel time can be affected by traffic patterns, Fu et al. (2003) present a hybrid predictive controller in which holding and stop-skipping control actions are taken each time a vehicle arrives at a stop. The problem is solved by means of genetic algorithms in which holding time may only take some discrete values. Chen et al. (2005) use a Kalman filter to predict arrival time by taking into account the effect of schedule recovery. Walker et al. (2005) is the first contribution where the crew rescheduling and timetable rescheduling problems are tackled an integrated way. An integer programming model with two distinctive blocks is developed in order to simultaneously construct a train timetable and crew roster, searching for minimizing the deviation from the original schedule. The problem is solved by an LP relaxation with column generation and branch-and-bound, and the model is tested on the Wellington Metro line. D'Ariano et al. (2007) introduce an optimization algorithm, based on a truncated branch-and-bound scheme, for finding a conflict-free schedule in a complex railway network. The objective function consists of minimizing the maximum delay for all trains when all stations are visited. A computational experiment is carried out on the Schiphol dispatching area of the Dutch railway network.

Burdett and Kozan (2009) have recently characterized this problem as a hybrid job shop scheduling problem with time windows. In most cases, all these strategies are conceived at the planning stage, but there are also approaches meant to be applied in real time. Mannino and Mascis (2009) solve the traffic control problem by means of an implicit enumeration of all feasible line runs that are assumed as instances of the classical representation of the job-shop scheduling problem. Computational tests are applied to the Milan metro network system. Corman et al. (2012) formulate the problem of finding a set of feasible schedules with 
a good trade-off between two objectives: the minimization of train delays and the maximization of passenger transfer connections. Test instances are based on the Dutch railway network around the main station of Utrecht. Louwerse and Huisman (2014) develop a model to determine a disposition timetable specifying which trains will still be operated given a disrupted infrastructure situation. The main objective is to maximize the passengers' service level.

In normal RTS operational circumstances, trains follow a periodic scheduling or a regular timetable. In the first case, trains departs from each station following a cyclic timetable, for example, they may be scheduled to depart at 7, 24 and 46 minutes every hour. In the regular case trains depart from the origin station at regular intervals. In both cases, when vehicles arrive at the last station, they repeat the same itinerary in the opposite direction. Periodic scheduling, based on the formulation of Serafini and Ukovich (1989), has been widely investigated by other authors like Chierici et al. (2004), Liebchen and Möhring (2002), and Liebchen and Peeters (2009), especially in the metropolitan railway context. Periodic timetables are easily memorized by users and can be computed with low effort (Wardman et al. 2004). Since in RTS regular timetabling trains are considered to successively run both directions of each line, an approach that simultaneously models two directions is required. However, modeling and solving the double direction problem to exclusively determine frequencies when track sharing and safety conditions have not been explicitly considered leads to solutions that cannot be easily implemented in terms of departure and arrival times.

The purpose of this paper is to develop a model capable of dealing with transport demand increments in a two-way line by means of inserting special short-turning services in an efficient manner. To this end, we formulate a model for the integration of short-turning services in order to obtain higher frequencies in some segments while maintaining the timetable of the previously programmed services. For a given demand, the origin and destination of shortturning services, departure and arrival times and dwell times are obtained by means of an optimization model that can be used at the tactical planning level. The proposed model is illustrated by an application to line $\mathrm{C} 5$ of the Madrid commuter railway system.

The remainder of this work is organized as follows. Section 2 describes the problem characteristics, as well as different assumptions. Section 3 presents a step by step formulation of linear optimization models and specifies the main contributions of the paper. In Sect. 4, the model is applied to a case study based on data from Line C5 of the commuter trains network in Madrid. Conclusions follow in Sect. 5.

\section{Problem statement}

The transit system considered in this paper consists of a set of two-way lines. For each line, trains follow a loop running from an origin station to a destination station, and back. In this kind of system, from a timetabling point of view, each line can be analyzed separately. We will therefore study the problem for a generic line. The proposed approach can then be applied to each one of the lines independently with some additional considerations about rolling stock. For the sake of simplicity, the start-to-end direction will be referred as the "up" direction and the opposite one as the "down" direction. The index $u$ will be used to represent data and variables corresponding to services in up direction, and the index $d$ will denote data and variables in the opposite direction.

Throughout this paper, we consider the following assumptions unless stated otherwise:

1. A two-way line with a track for each direction, i.e. a one-way loop network.

2. We consider the situation in which a set of trains are already operating on the line following a regular timetable. Note that if the two first services in both ends of the line 


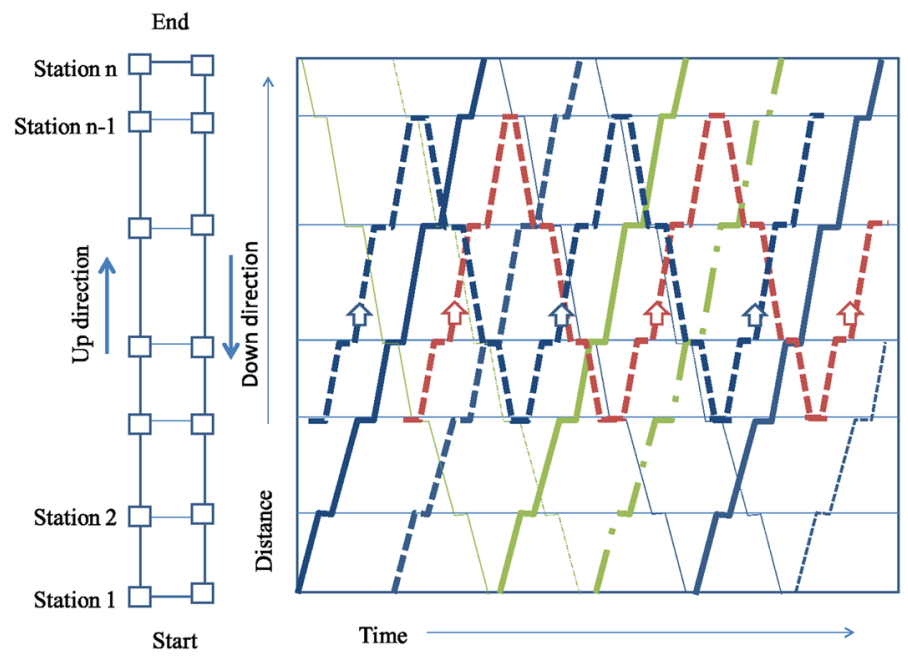

Fig. 1 Short-turning services with the same headway as full-length services

are launched exactly at the same instant and the rest of trains are launched considering a constant inter-departure interval, a symmetrical regular timetable is obtained. The latter situation is usually the easiest and best option to achieve regular frequencies in both directions (see Liebchen 2004).

3. We consider that an increase in the demand corresponding to certain origin-destinations pairs should be dealt with. These demand peaks could respond to known in advance exogenous events, e.g. football matches, demonstrations, major fairs or exhibitions, etc., and will usually require an increase in supply between important stations (i.e. transport interchange facilities).

4. We assume that the original timetables are optimally designed from the operator's point of view, and can satisfy the passenger demand in normal circumstances i.e., original timetables have the smallest number of trains that enable the operator to satisfy all the original demand. So, the inclusion of short-turning services must respond exclusively to the extra increase in demand.

5. We assume the existence of short-turning facilities at some stations.

6. At each station and in both directions, we assume the existence of a large enough number of tracks to place simultaneously different trains when they are stopped. This allows overtaking and overlapping at stations.

7. Empty movements of short-turning trains from engine sheds to turn-back points are not considered. These empty movements should be scheduled separately from the shortturning services timetable. Usually, these trips have a lower priority than the ordinary services, and can be easily incorporated in the existing schedule, sometimes by simple inspection. This analysis is out of the scope of this work but we refer the interested reader to Abril et al. (2004), Cacchiani et al. (2010a), Canca (2009) and Flier et al. (2009).

Figure 1 illustrates a regular symmetric timetable where two trains are running in each direction and two short-turning service are running between stations 3 and $n-1$ with the same headway as for the regular services. The horizontal axis represents the time and the vertical one the distance from the origin station. The cycle length of a shuttle is defined as the time needed to complete a loop. It is a direct consequence of the route length and the train 


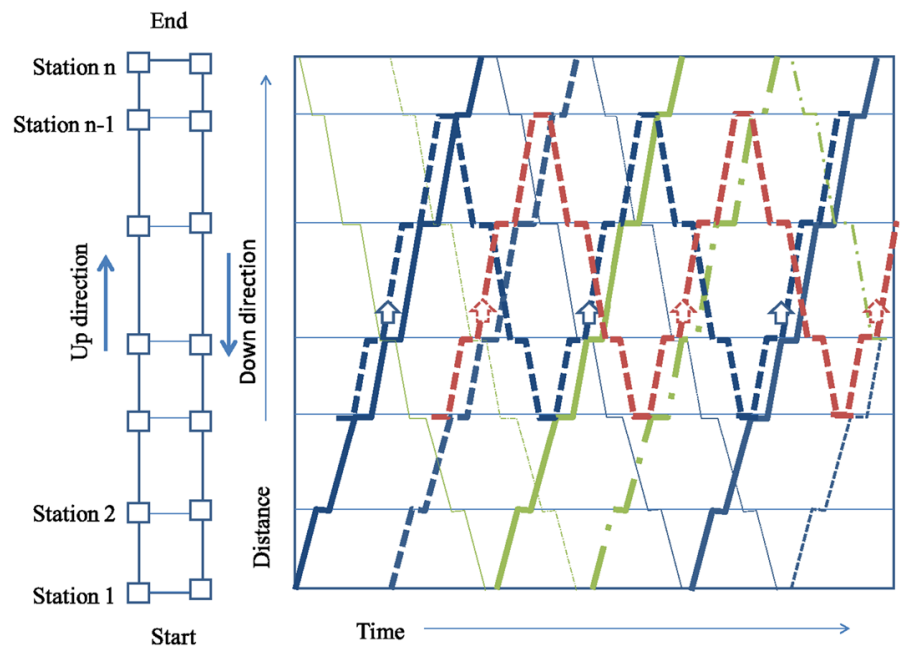

Fig. 2 A little offset of short-turning services to capture a high fraction of demand

speed. So, shuttle insertions into a previously defined timetable normally lead to schedules with non-regular frequencies unless speeds and headways follow certain predefined rules, according to Vijayaraghavan and Anantharamaiah (1995).

In order to become effective, short-turning services should capture a certain fraction of the demand between their origin and destination. As a consequence, they should pass every station a certain time before the regular (full-length) service (see Fig. 2). Precisely, this relative offset determines the success of the short-turning service. Note that if the offset is high, passengers coming to the stations in the inner zone after the short-turning service departure can take the regular service to reach their destination, thus contributing to a higher occupancy of regular services. On the other hand, when the headway of regular services is high, some passengers can also take the short-turning trip even if their destination is outside the inner zone, giving rise to an undesirable phenomenon called induced transfers at the short-turning origin and destination stations (Delle Site and Filippi 1998).

Designing the best timetable to satisfy the increase in the demand defined by an origindestination increment matrix can be decomposed into two different but related decision problems. The first is the selection of the zone (origin and destination) of short-turning services, which is usually analyzed specifically for each situation following a trial and error procedure. The second decision concerns the design of the timetable, i.e. how to adequately insert the new services into the existing ones, determining departure and arrival times. Here, both the zone selection and the timetable design are studied simultaneously by means of a mixed integer non-linear optimization model.

\section{Mathematical model}

In order to describe the optimization model, in the following, parameters and variables will be introduced. Let $S=\{1, \ldots, n\}$ be the set of stations along the line, numbered following the up direction, let $M=\{1,2, \ldots, m\}$ be the set of train services, and let $L=\{(1,2), \ldots$, $(n-1, n)\}$ be the set of segments linking consecutive stations. Let $h_{\min }$ be the minimum headway between train services. 


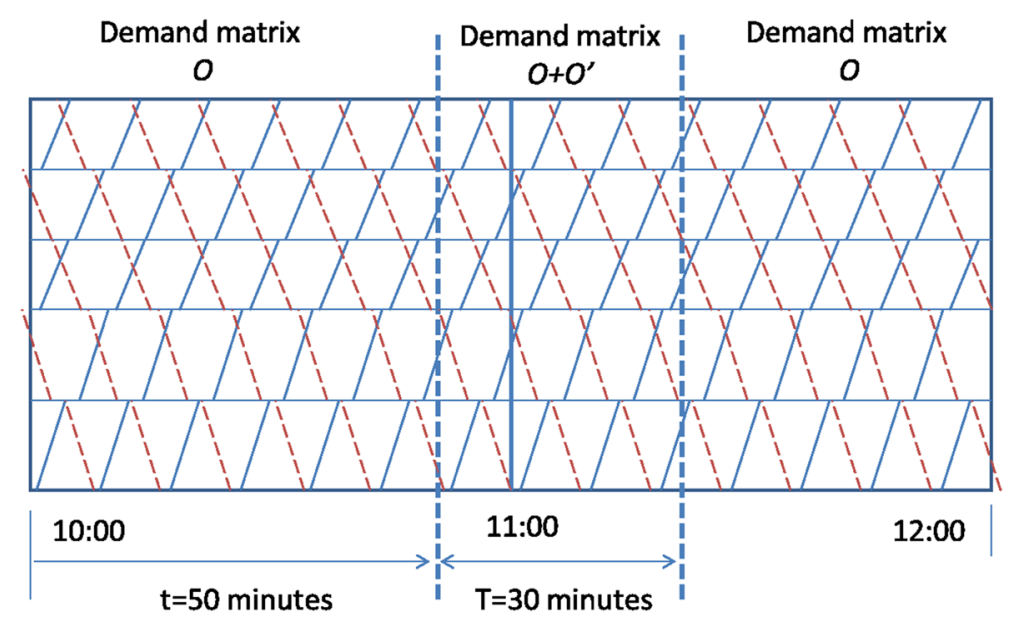

Fig. 3 Example of a demand increment scenario

Full-length services go from station 1 to $n$ and vice versa, and stop at every station in between. These services, as well as their regular timetables are given, and are sufficient to satisfy the original demand matrix $O$ during a certain time interval $\Delta$. An increment of the demand is given by a matrix $O^{\prime}$ and occurs during a certain disturbance time interval $T \subseteq \Delta$. The problem consists of selecting a pair of stations among those with short-turning facilities $p, r \in S, 1 \leq p<r \leq n$, and inserting a certain number of short-turning services between them in order to satisfy the increment of the demand, while ensuring feasibility.

Before defining the optimization model, we describe the demand matrices, and explain how to consider the load and capacity of train services, the headway between them and between short-turning and full-length services, and the short-turning fleet size.

\subsection{Demand matrix and increment of demand}

Let $O$ be the original origin-destination matrix defining movements between each pair of stations on the line during the time interval $\Delta$. The elements of $O$ are denoted by $d_{i j}$ where $i, j \in S$. Let $O^{\prime}$ the origin-destination matrix defining an increment of demand during the time interval $T \subseteq \Delta$. The elements of $O^{\prime}$ are denoted by $d_{i j}^{\prime}$, where $i, j \in S$. Figure 3 depicts an example where $\Delta=[10: 00,12: 00]$ and $T=[10: 50,11: 20]$.

\subsection{Loads and capacity}

The original load of passengers over the segment $(k, k+1)$ during the disturbance interval $T \subseteq \Delta$ (i.e., the number of passengers who traverses segment $(k, k+1)$ during interval $T$ ) can be obtained by applying a proportion factor to the load of passengers over segment $(k, k+1)$ during the time interval $\Delta$. The following equations describe the loads of passengers who respectively follow the up and down directions during the disturbance interval:

$$
q^{k u}=\sum_{i, j \in S: i \leq k<j} d_{i j} \frac{T}{\Delta}, \quad \forall k \in L,
$$




$$
q^{k d}=\sum_{i, j \in S: j \leq k<i} d_{i j} \frac{T}{\Delta}, \quad \forall k \in L .
$$

Let $Q$ be the maximum load over the segment set irrespective of the direction of travel, that is, $Q=\max _{z \in\{u, d\}}\left\{\max _{1 \leq k \leq L} q^{k z}\right\}$.

Since we assume the existence of a regular timetable that satisfies the original demand defined by matrix $O$ with the smallest possible number of trains, if train units have a capacity of $C a p$ passengers, then the initial headway $h$ holds:

$$
\frac{Q}{C a p}=\frac{\Delta}{h}=\text { freq. }
$$

Note that this equation implies the existence of a proportionality constant which transforms load capacities into operation times (proportionality law). Additionally, the loads resulting from the increase of the demand matrix $O^{\prime}$ over segment $(k, k+1)$ are:

$$
\begin{aligned}
q^{\prime k u} & =\sum_{i, j \in S: i \leq k<j} d_{i j}^{\prime}, \quad \forall k \in L, \\
q^{\prime k d} & =\sum_{i, j \in S: j \leq k<i} d_{i j}^{\prime}, \quad \forall k \in L .
\end{aligned}
$$

During the perturbation interval, the total load for each segment $(k, k+1)$ in each direction is the sum of the original load and of the load due to the demand increment:

$$
\begin{aligned}
& \bar{q}^{k u}=q^{k u}+q^{\prime k u}=\sum_{i, j \in S: i \leq k<j}\left(d_{i j} \frac{T}{\Delta}+d_{i j}^{\prime}\right), \quad \forall k \in L, \\
& \bar{q}^{k d}=q^{k d}+q^{\prime k d}=\sum_{i, j \in S: j \leq k<i}\left(d_{i j} \frac{T}{\Delta}+d_{i j}^{\prime}\right), \quad \forall k \in L .
\end{aligned}
$$

Suppose a short-turning zone is defined between two stations $p, r \in S, 1 \leq p<r \leq n$, both included. So, the potential numbers of passengers over every segment $(k, k+1)$ within the short-turning zone considering both directions are:

$$
\begin{aligned}
& \bar{q}_{p r}^{k u}=\sum_{i, j \in S:}\left(d_{i j} \frac{T}{\Delta}+d_{i j}^{\prime}\right), \quad p, r \in S ; k \in L: p \leq k \leq r-1, \\
& \bar{q}_{p r}^{k d}=\sum_{i, j \in S:}\left(d_{i j} \frac{T}{\Delta}+d_{i j}^{\prime}\right), \quad p, r \in S ; k \in L: p \leq k \leq r-1 .
\end{aligned}
$$

However, short-turning services cannot capture all the demand inside the short-turning zone. In fact, a fraction of the passengers in the inner zone will use full-length services to travel inside the short-turning zone. Applying again the proportionality law, this fraction will be a consequence of the length of the offset between a full-length service and the short-turning service that precedes it. Figure 4 depicts this situation for an example with three short-turning services. Solid bold lines represent regular (full-length) services running with headway $h$. Dashed lines represent short-turning services running with headway $h^{\prime}$. The offset between short-turning and full-length services is denoted by $f$. Note that several conditions on $f$ and $h^{\prime}$ can be deduced from Fig. 4, which will be explained in Sect. 3.3.

The inclusion of the variable $f$ achieves two results: first a separation of passengers into those who are served by full-length services and those who are served by short-turning services, and secondly, $f$ directly determines timetables for these new trains. If we suppose a 


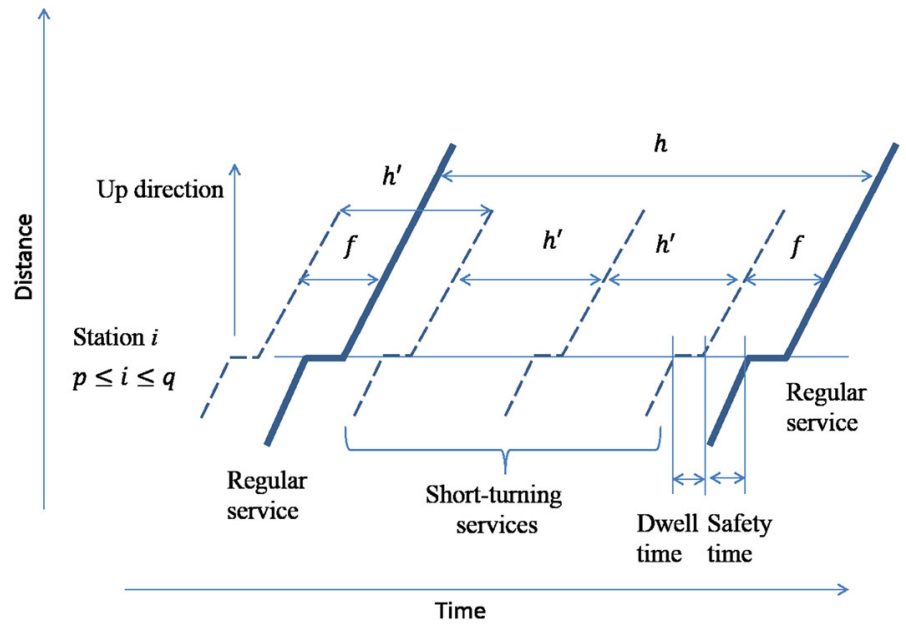

Fig. 4 Offset, headway and short-turning headway

regular rate of passenger arrivals at stations, then passengers arriving after last short-turning service traveling inside the short-turning zone will take the full-length service to reach their destination. Therefore, for a given zone defined by stations $p, r \in S$, the residual load pattern (passenger load for each segment over the full length zone) is obtained as follows:

$$
\hat{q}_{p r}^{k z}= \begin{cases}\bar{q}^{k z}, & p, r \in S ; k \in L: k<p \text { or } k>r ; z=u, d, \\ \bar{q}^{k z}-\frac{(h-f)}{h} \bar{q}_{p r}^{k z}, & p, r \in S ; k \in L: p \leq k \leq r ; z=u, d .\end{cases}
$$

Note that, once variable $f$ has been determined, $\bar{q}^{k z}$ and $\bar{q}_{p r}^{k z}$ can be directly computed from the elements of $O$ and $O^{\prime}$. The segments outside the short-turning zone maintain the same load, whereas inside the zone delimited by stations $p$ and $r$, the load for full-length services diminishes by $\frac{(h-f)}{h} \bar{q}_{p r}^{k z}(z=u, d)$. This is precisely the number of passengers who take the short-turning services in the inner zone. In any case, feasible solutions have to respect capacity constraints. So, for full-length services, the following bounds must hold:

$$
\frac{h}{T} \hat{q}_{p r}^{k z} \leq \text { Cap, } \quad \forall p, r \in S ; k \in L ; p \leq k \leq r ; z=u, d .
$$

Similarly for short-turning services, considering that the capacity is the same as for full-length ones, the following constraints must hold:

$$
\frac{h^{\prime}}{T} \frac{(h-f)}{h} \bar{q}_{p r}^{k z} \leq \text { Cap, } \quad \forall p, r \in S ; k \in L ; z=u, d .
$$

A different capacity may also be considered, which is treated as a variable according to the rolling-stock possibilities (Canca et al. 2014). In fact, for some larger increments of demand, increasing short-turning capacity yields feasible solutions. Note that the set of constraints (2) need to hold only for the selected location of turn-back points. Figure 5 illustrates the effect of a zone definition over the full-length passenger load. In particular, the full-length load pattern in normal circumstances (dashed line) and the pattern after the demand increment are shown. Figure 6 depicts the demand captured by short-turning services and the residual demand for the full-length pattern. Note that the residual full-length load pattern exceeds the original one 


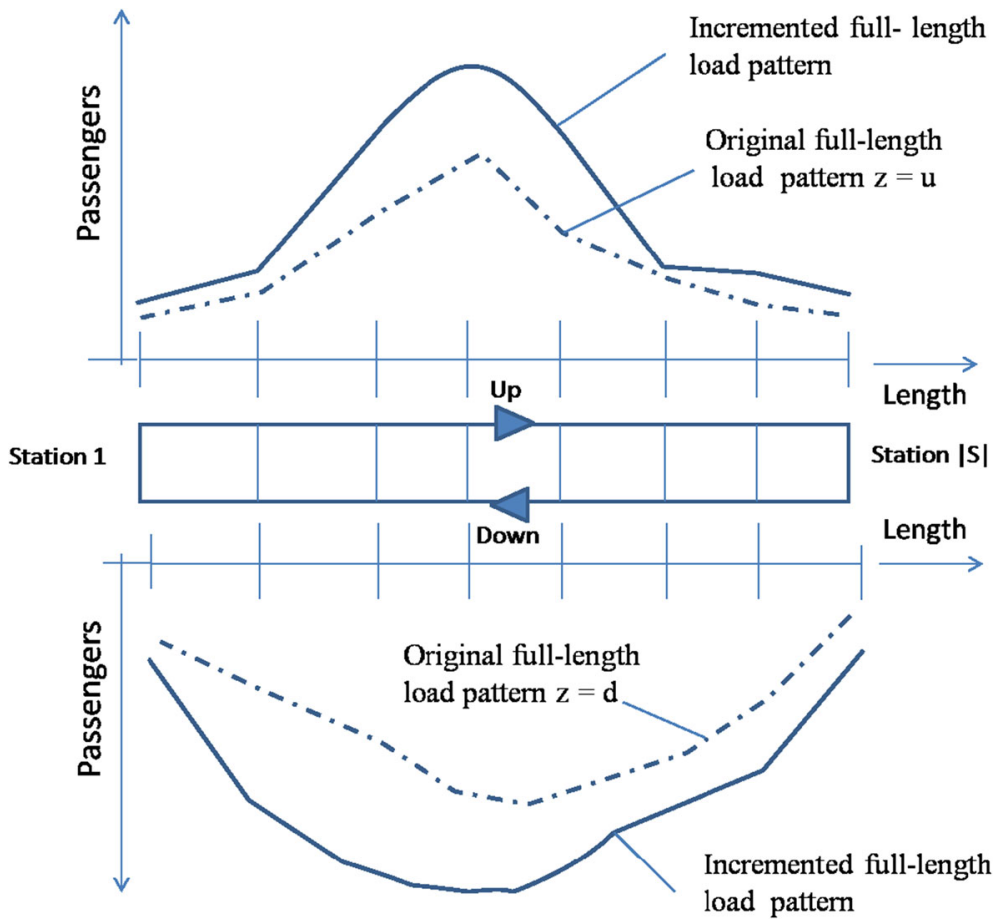

Fig. 5 Effect of a demand increment over the full-length original load pattern

(without demand increment). This phenomenon is a consequence of the short-turning offset, i.e., passengers arriving after a short-turning service departure can take the next full-length service if it arrives before next short-turning service.

\subsection{Offsets and short-turning headway}

Several conditions on $f$ and $h^{\prime}$ can be deduced from Fig. 4. In order to ensure regularity, the number of short-turning services should be a multiple of the number of full-length services. Let $N$ be the number of short-turning services between two full-length ones, then the headways $h$ and $h^{\prime}$ satisfy $h=N h^{\prime}$, and $f \leq h^{\prime}$. The offset should also be larger than the minimum headway $h_{\text {min }}$, that is, $h_{\text {min }} \leq f$.

If we consider a constant dwell time $d>0$ for all the services, then each short-turning service will stop $d$ minutes at each station. Moreover, the next service should arrive at least $h_{\text {min }}$ minutes after the departure of the last train. As a consequence, the maximum number of short-turning services is bounded as follows: $N\left(d+h_{\min }\right)+h_{\min } \leq h$. These expresions act as constraints in the optimization model proposed in Sect. 3.7.

\subsection{Fleet size}

The capacity constraints derived from Eqs. (1) and (2) are zone-dependent. The model must determine the location of turn-back points, and this decision heavily influences the shortturning fleet size. Therefore, the problem now consists of detecting the most convenient pair of stations to define the short-turning service operating zone according to the expected 


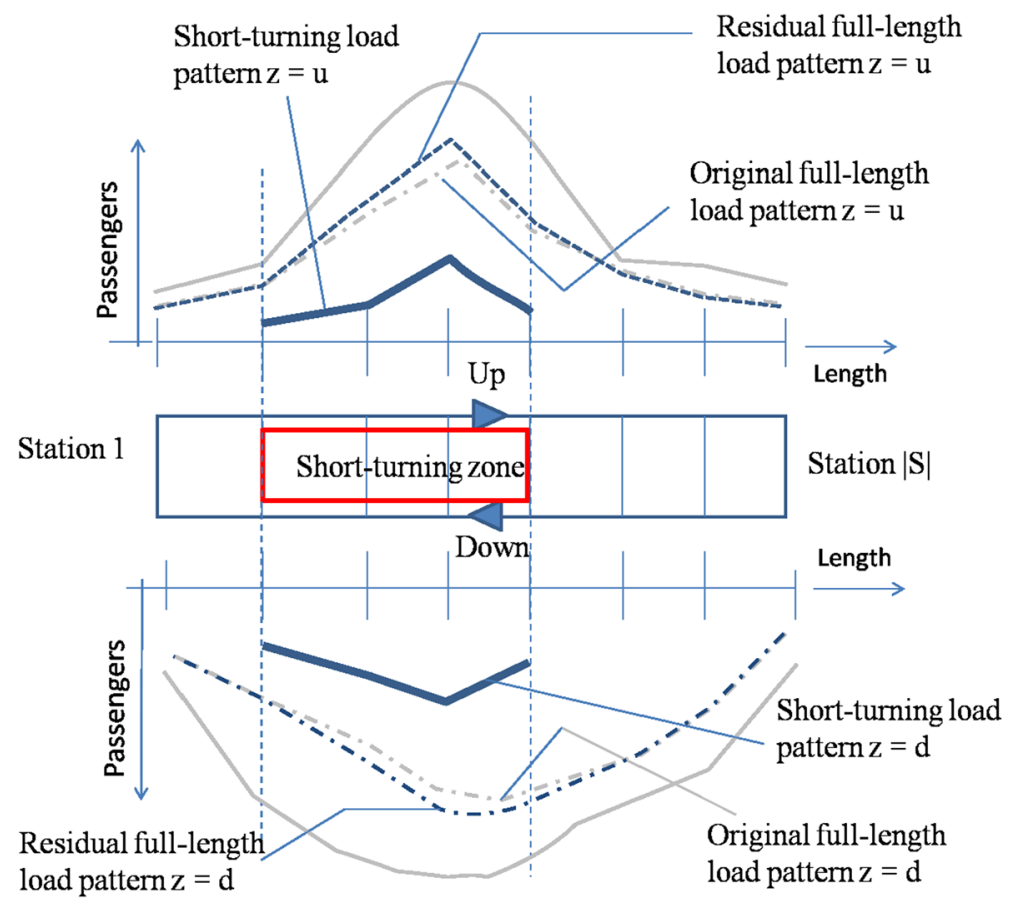

Fig. 6 Effect of short-turning over full-length total load pattern

increment of the demand. Given a pair of stations $p, r \in S, p<r$ and a known average train speed $v$, the cycle length for a service running from $p$ to $r$ is

$$
C_{p r}=\frac{2}{v} \sum_{m \geq p}^{r-1} L_{m}+2(r-p) d,
$$

and the required number of train units will be

$$
F_{p r}=\left\lceil\frac{C_{p r}}{h^{\prime}}\right\rceil,
$$

where $\lceil$.$\rceil represents the next larger integer.$

The larger the speed of the train unit, the smaller the cycle for each origin destination pair. Obviously, the train speed is bounded by safety and technical aspects. Without loss of generality we will consider the same speed on each segment. However, speed dependency on segments, directions, as well as different dwell times at stations, can be easily introduced in the expression (3) by accumulating running and dwell times segment by segment.

On the entire line, assuming train units run with the same speed, if all the services begin their journey at station 1 , the number of trains operating according to the regular service in normal circumstances (i.e. without demand increment) will be

$$
F_{1|S|}=\left\lceil\frac{C_{1|S|}}{h}\right\rceil .
$$

Now consider a limited fleet size, a parameter $F_{\max }$, to operate on the line, assuming that the short-turning zone is defined between stations $p$ and $r$. Then the following constraint must 
be satisfied:

$$
\left\lceil\frac{C_{1|S|}}{h}\right\rceil+\left\lceil\frac{C_{p r}}{h^{\prime}}\right\rceil \leq F_{\text {max }} .
$$

Alternatively, if we consider only a limited reserve fleet size to operate the line, a parameter $F_{\text {res }}$, then

$$
\left\lceil\frac{C_{p r}}{h^{\prime}}\right\rceil \leq F_{\text {res }}
$$

As already mentioned, high demand increments suggest lower short-turning headways, which leads to an increase in fleet size. On the other hand, ceteris paribus, a long running cycle supposes a larger fleet size. So, the selection of turn-back points will influence both demand reduction over the full-length line and the short-turning fleet size.

\subsection{Zone selection}

In the last three subsections, we have presented the variables and constraints of the model. Note that capacity and fleet size constraints are formulated under the assumption that a certain zone has previously been defined. We now define a set of binary variables in order to perform the most convenient zone selection. The constraints are modified accordingly to include these variables.

Let $S^{\prime} \subset S$ be a subset of stations containing short-turning facilities. Let $\delta_{p r}$ be a binary variable equal to one if a zone between stations $p, r \in S^{\prime}: p<r$, is selected for short-turning services. Then, the constraint

$$
\sum_{p, r \in S^{\prime}: p<r} \delta_{p r}=1 .
$$

ensures the selection of only one zone. For this zone, capacity and fleet constraints must hold. These conditions can be modeled by considering a sufficiently large constant $M$, and by including the corresponding binary variables in the appropriate constraints as follows:

1. Full-length services outside the short-turning zone:

$$
\begin{aligned}
& \frac{h}{T} \bar{q}^{k z} \leq C a p+M\left(1-\delta_{p r}\right), \\
& \quad p, r \in S^{\prime}: p<r ; k \in L: k<p \text { or } k>r ; z=u, d .
\end{aligned}
$$

2. Full-length services inside the short-turning zone:

$$
\begin{aligned}
& \frac{h}{T}\left(\bar{q}^{k z}-\frac{(h-f)}{h} \bar{q}_{p r}^{k z}\right) \leq \text { Cap }+M\left(1-\delta_{p r}\right), \\
& \quad p, r \in S^{\prime}: p<r ; k \in L: p \leq k \leq r ; z=u, d .
\end{aligned}
$$

3. Short-turning services:

$$
\begin{aligned}
\frac{h^{\prime}}{T} \frac{(h-f)}{h} \bar{q}_{p r}^{k z} \leq C a p+M\left(1-\delta_{p r}\right), \\
p, r \in S^{\prime}: p<r ; k \in L: p \leq k \leq r ; z=u, d .
\end{aligned}
$$

\subsection{Objective function}

Two different objectives functions are proposed to determine the most convenient shortturning definition and trains timetables. The first one consists of the maximization of passengers carried by short-turning services. Since the short-turning services capture a fraction $(h-f) / h$ of the total demand inside the corresponding short-turning zone, given a pair $p$, 
$r$ of turn-back points, the number of passengers $\operatorname{Pas}(p, r)$ moved by short-turning services can be estimated as

$$
\operatorname{Pas}(p, r)=\frac{h-f}{h}\left[\sum_{i, j \in S: p \leq i<j \leq r}\left(\left(d_{i j}+d_{j i}\right) \frac{T}{\Delta}+d_{i j}^{\prime}+d_{j i}^{\prime}\right)\right],
$$

where up and down directions are considered. Then, multiplying the demand for each possible zone by the binary activation variable and summing up for all zones, we obtain a first objective function $(O F 1)$, which maximizes the short-turning captured demand:

$$
\text { (OF1) Maximize } \frac{h-f}{h}\left[\sum_{p, r \in S^{\prime}: p<r} \operatorname{Pas}(p, r) \delta_{p r}\right] \text {. }
$$

Despite the fact that this objective function makes sense, some undesired effects can occur when the zone is selected by applying this criterion. Supposing that a certain value of $f$ has already been determined, the objective function will try to maximize the number of passengers captured by short-turning services. Adjustments of measure units would imply to add other criteria to the problem such as assignation of a cost for the passenger load and fleet size. Since the number of passengers typically increases with the size of the zone, larger zones will be preferable. The model will then try to select turn-back points located as far as possible. However, long short-turning zones lead to larger cycle times and, as a result, to larger fleet sizes. For this reason, a fleet limitation constraint should be added and, in this situation and depending on the line topology (length of segments), the solution will propose the most extensive zone while respecting fleet size limitation even if train occupancy levels are low. This inconvenience can be removed by maximizing the occupancy of each short-turning train. Then, for our next objective function $\left(O F 1^{\prime}\right)$, the total demand over the planning horizon $T$ is divided by the fleet size $(F S)$ needed to provide the services:

$$
\left(O F 1^{\prime}\right) \text { Maximize } \frac{1}{F S} \frac{h-f}{h}\left[\sum_{p, r \in S^{\prime}: p<r} \operatorname{Pas}(p, r) \delta_{p r}\right] .
$$

The variable $F S$ is logically related with each possible zone by means of the constraint

$$
F S=\left\lceil\frac{1}{h^{\prime}}\left[\sum_{p, r \in S^{\prime}: p<r} C_{p r} \delta_{p r}\right]\right\rceil \text {. }
$$

Moreover, the following condition must hold:

$$
1 \leq F S \leq F_{\text {res }} .
$$

This way, the zone selection procedure takes into account the length of the zone as well as the convenience of capturing as many passengers as possible. It can be observed that $\left(O F 1^{\prime}\right)$ is a non-linear function and that the numerator and denominator are not expressed in the same units and on the same scale. Some adjustments would therefore be needed in order to use this objective function. The weights used when considering these aspects influence the resulting solution and, to be consistent, would force a multiobjective analysis. Instead, we take into account the nature of the problem and propose a simple linear function which takes advantage of it. The full-length services are already occupied and working under profitable conditions without the demand increment. So, after the demand increment, our aim then is to equilibrate the occupancy of the new short-turning services with the existing ones. This 


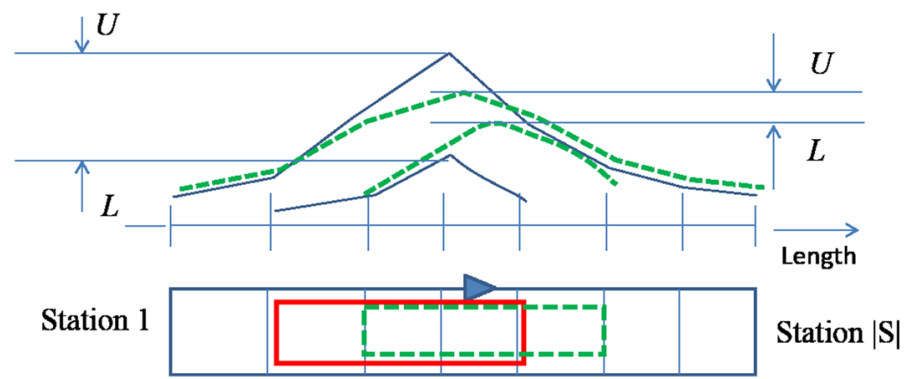

Fig. 7 Zone selection mechanism by maximizing equilibrium of loads

objective implies the minimization of the difference between passengers load for both kind of services in the selected zone. In fact, since the demand distribution along the line can be extremely variable, it is necessary to consider the full-length demand along the entire line. This attempt of equilibrating occupancy may be modeled by minimizing the difference between an upper bound $U$ and a lower bound $L$ on the full-length and short-turning loads respectively (see Fig. 7). In this way, minimizing $U$ along the line will help revert the situation to the original one, moving the excess of demand from full-length services to short-turning ones. On the other hand, given several candidate solutions for achieving this goal, we select the more convenient zone maximizing $L$, i.e., the origin and end of short-turn movements are selected so that the load difference between full-length and short-turning units becomes minimal.

Let $M^{\prime}$ be a positive large enough constant, then the following inequalities must hold:

1. Bounding full-length services outside the short-turning zone:

$$
\begin{aligned}
\frac{h}{T} \bar{q}^{k z} & \leq U+M^{\prime}\left(1-\delta_{p r}\right), \\
p, r & \in S^{\prime}: p<r ; k \in L: k<p \text { or } k>r ; z=u, d .
\end{aligned}
$$

2. Bounding full-length services inside the short-turning zone:

$$
\begin{gathered}
L-M^{\prime}\left(1-\delta_{p r}\right) \leq \frac{h}{T}\left(\bar{q}^{k z}-\frac{(h-f)}{h} \bar{q}_{p r}^{k z}\right) \leq U+M^{\prime}\left(1-\delta_{p r}\right), \\
p, r \in S^{\prime}: p<r ; k \in L: p \leq k \leq r ; z=u, d .
\end{gathered}
$$

3. Bounding short-turning services:

$$
\begin{array}{r}
L-M^{\prime}\left(1-\delta_{p r}\right) \leq \frac{h^{\prime}}{T} \frac{(h-f)}{h} \bar{q}_{p r}^{k z} \leq U+M^{\prime}\left(1-\delta_{p r}\right), \\
p, r \in S^{\prime}: p<r ; k \in L: p \leq k \leq r ; z=u, d .
\end{array}
$$

\subsection{Disjunctive model}

According to the second optimization criterion discussed in Sect. 3.6, the objective function will simply minimize the difference between $U$ and $L$ : 
(OF2) Minimize $U-L$

s.t. constraints (4)-(10) and

$$
\begin{aligned}
& h=N h^{\prime} \\
& h_{\text {min }} \leq f \leq h^{\prime} \\
& N\left(d+h_{\text {min }}\right)+h_{\text {min }} \leq h \\
& \frac{2}{v} \sum_{m \geq p}^{r-1} L_{m}+2(r-p) d \leq h^{\prime} F_{\text {res }}+M^{\prime \prime}\left(1-\delta_{p r}\right), \\
& \quad p, r \in S^{\prime}: p<r \\
& L, U, h^{\prime}, f \geq 0 \\
& \delta_{p r} \in\{0,1\} ; p, r \in S^{\prime}: p<r .
\end{aligned}
$$

Constraints (13)-(15) have already been explained in Sect. 3.3. Constraints (16) are similar to (3) but include turn-back points location. If $\delta_{p r}=1$, the required fleet size should be less than or equal to $F_{r e s}$; otherwise, no fleet size limitation is imposed.

\section{Illustration}

In order to illustrate the performance of the optimization model, we have considered a corridor with an approximate length of $20 \mathrm{~km}$ composed of 10 stations. The corridor is a piece of the C5 line of the Madrid commuter railway system. We have used an initial origin-destination matrix $O$ with more than 9,000 trips corresponding to a one-hour interval $\Delta$.

$$
O=\left(\begin{array}{llllllllll}
0 & 25 & 40 & 45 & 65 & 50 & 85 & 205 & 20 & 10 \\
35 & 0 & 35 & 40 & 55 & 40 & 75 & 175 & 20 & 10 \\
200 & 175 & 0 & 65 & 85 & 65 & 115 & 275 & 30 & 15 \\
10 & 5 & 10 & 0 & 120 & 95 & 165 & 385 & 40 & 20 \\
115 & 100 & 135 & 1,750 & 0 & 750 & 1,325 & 3,120 & 335 & 175 \\
160 & 140 & 180 & 2,435 & 655 & 0 & 1,335 & 3,150 & 335 & 180 \\
150 & 135 & 170 & 2,270 & 610 & 595 & 0 & 2645 & 280 & 150 \\
25 & 25 & 30 & 395 & 105 & 105 & 395 & 0 & 260 & 140 \\
15 & 15 & 15 & 230 & 60 & 60 & 230 & 25 & 0 & 25 \\
5 & 5 & 5 & 75 & 20 & 20 & 75 & 10 & 15 & 0
\end{array}\right)
$$

This matrix represents the typical inbound pattern of a railway line where the majority of passenger movements are near the central district of a city, which in this case consists of stations 5, 6 and 7. This is depicted in Fig. 8. The horizontal axis represents the stations and the vertical axis the train occupancy at each station. The up direction has been arbitrarily

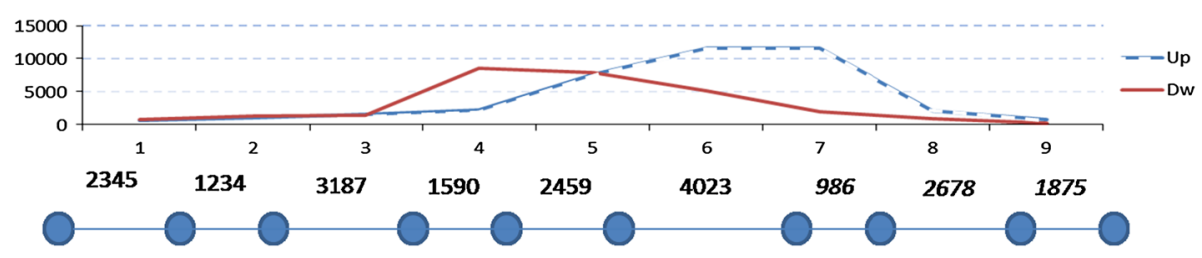

Fig. 8 Initial load profiles 


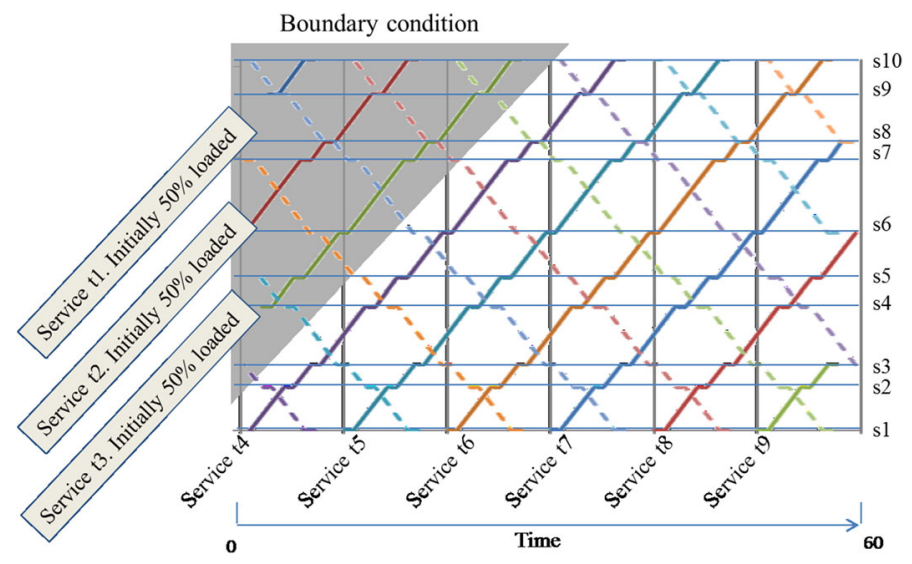

Fig. 9 Initial timetable and boundary condition (up direction)
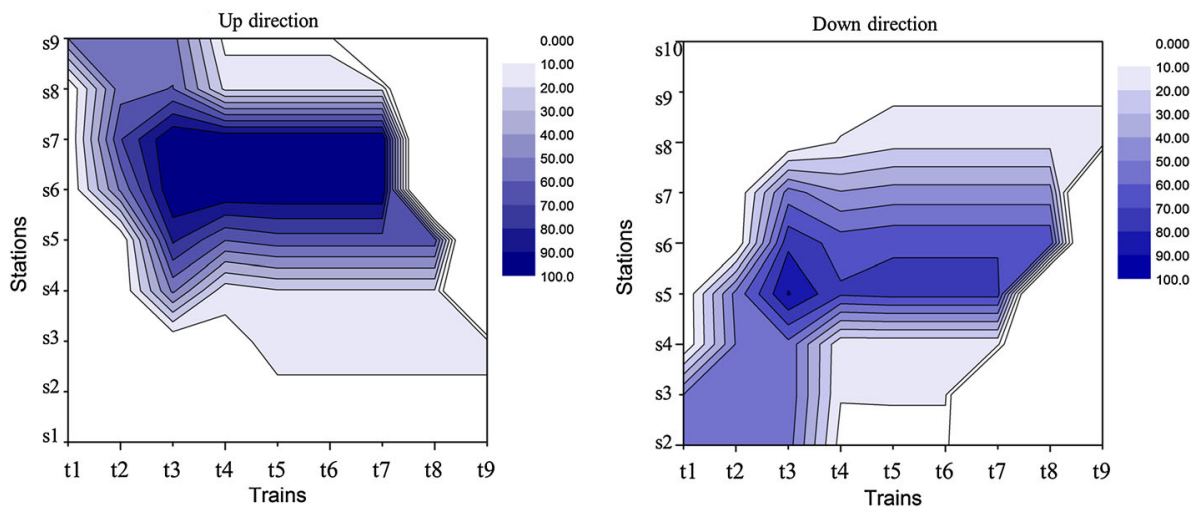

Fig. 10 Occupancy map corresponding to the initial timetable and demand matrix $O$

defined from station 1 to station 10. The length of the segments is measured in meters, and is provided over each pair of consecutive stations in the figure.

For these initial demand conditions, we have obtained a first regular timetable capable of serving to the most loaded segment (Grosfeld-Nir and Bookbinder 1995). This timetable was built considering a headway of 10 minutes for services in both directions (see Fig. 9). We have assumed the existence of short-turning facilities in a subset of stations, namely stations 2, 3, 6, 7, 8. Given the timetable, we have simulated the regular timetable in order to obtain vehicle occupancies and passenger waiting times. This simulation was performed using pyRsim, a free timetable simulator written in python by the authors and available at http://dco.us.es/pyRsim. The python script performs a simulation considering passenger boarding and alighting at each station and determining the most convenient train capacity to serve the most loaded segment, as is normally done when developing regular timetables in practice. The resulting vehicle capacity in our experiment is equal to 1,900 passengers.

The results of the simulation are shown in Fig. 10. In this figure, we obtain an occupancy map for each direction. This map is the projection of train occupancy on each segment over the plane train services-distance. The vertical axis represents the distance from the first station, 


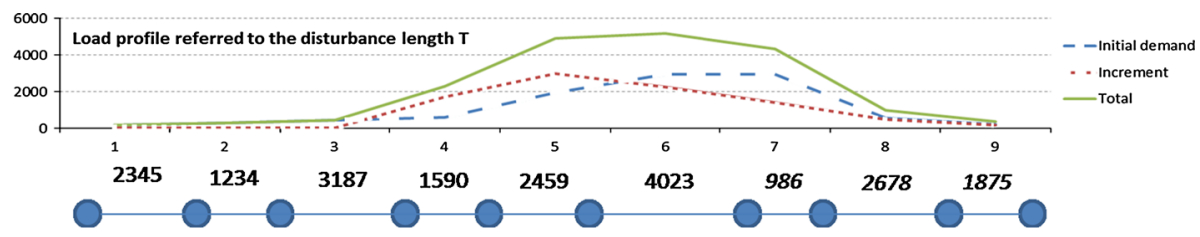

Fig. 11 Load profiles after imposing the increment of demand (Up direction)

and the horizontal axis represents the train service number. The graph on the left corresponds to the up direction, whereas the graph on the right maps the opposite direction.

Some considerations must be taken into account in order to understand these figures. Services are launched from the first or the last station (depending on the direction), starting at time $t=0$, every 10 minutes with a speed of $45 \mathrm{~km} / \mathrm{h}$ and a dwell time of one minute. The timetable is initially completed with trains departing from other stations to maintain a regular timetable along the entire planning horizon. As a consequence, if we depict the onehour timetable, we can see nine services. The first service launched from the origin station corresponds to service number 4, as it is depicted in Fig. 9. At time $t=0$, service number 1 (t1 in Fig. 10) departs from station 9, the second one (t2) departs from station 6 and the third one ( $\mathrm{t} 3$ ) from station 4 . The inclusion of these initial trains allows us to have a complete one-hour timetable while maintaining a certain temporal continuity. Moreover, as a boundary condition, trains beginning services from stations other than station 1 are initially loaded at $50 \%$ of their capacity (see Fig. 9).

Concerning the up direction, we note a high occupancy level between stations 6 and 7 for trains running services between $\mathrm{t} 3$ and $\mathrm{t} 7$. Moreover, this situation yields an average waiting time (AWT) of 6.66 minutes per passenger. In perfect conditions, if the capacity of trains was not reached in any segment, the AWT should be exactly half of the headway, that is five minutes. For the down direction, the largest occupancy occurs between stations 5 and 6 for services $\mathrm{t} 3$ to $\mathrm{t} 8$. In this direction, since the load is lower than in up direction, the resulting AWT is exactly five minutes. These results are consistent with the expectations we make after observing the load profiles of Fig. 8.

In order to overload the line, a peak origin-destination matrix $O^{\prime}$ corresponding to a disturbance length $T$ of 15 minutes was imposed over the corridor. For this experiment, the disturbance offset was fixed to 30 minutes, i.e. the beginning of new arrivals takes place at $t=30$. This matrix imposes an increment of demand in the outbound direction from stations 4 and 5 towards the rest of the line. This is typically the situation at the end of a demonstration or when a sporting event takes place at a city center.

$$
O^{\prime}=\left(\begin{array}{llllllllll}
0 & 0 & 0 & 0 & 0 & 0 & 0 & 0 & 0 & 0 \\
0 & 0 & 0 & 0 & 0 & 0 & 0 & 0 & 0 & 0 \\
0 & 0 & 0 & 0 & 0 & 0 & 0 & 0 & 0 & 0 \\
75 & 250 & 350 & 0 & 25 & 375 & 500 & 525 & 175 & 100 \\
150 & 400 & 500 & 25 & 0 & 325 & 375 & 400 & 125 & 50 \\
0 & 0 & 0 & 0 & 0 & 0 & 0 & 0 & 0 & 0 \\
0 & 0 & 0 & 0 & 0 & 0 & 0 & 0 & 0 & 0 \\
0 & 0 & 0 & 0 & 0 & 0 & 0 & 0 & 0 & 0 \\
0 & 0 & 0 & 0 & 0 & 0 & 0 & 0 & 0 & 0 \\
0 & 0 & 0 & 0 & 0 & 0 & 0 & 0 & 0 & 0
\end{array}\right)
$$

Figure 11 illustrates the new load profiles, referred to the disturbance length interval $T$, corresponding to the up direction. 

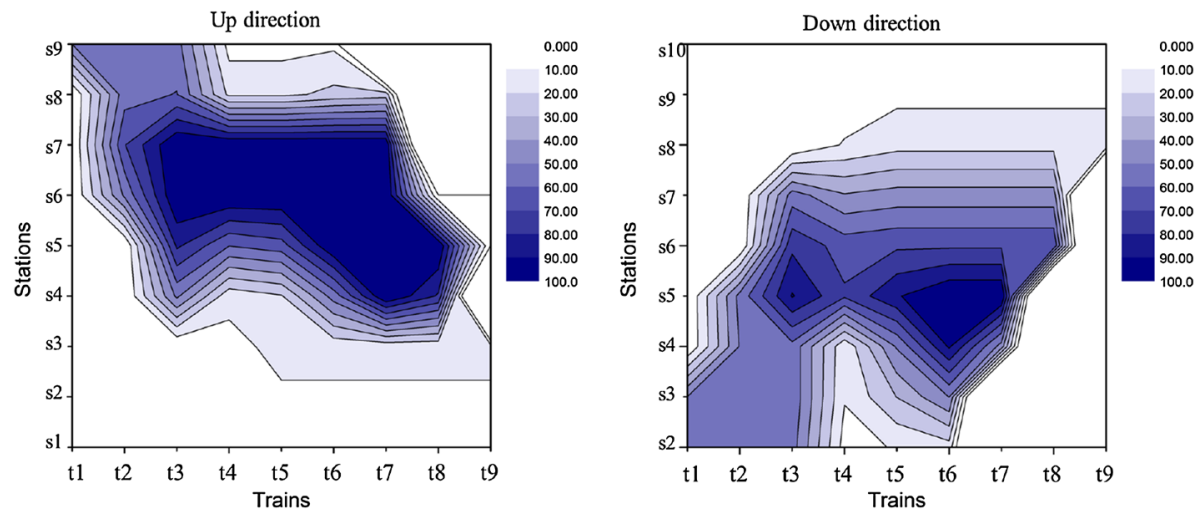

Fig. 12 Occupancy map corresponding to the initial timetable and demand matrix $O+O^{\prime}$

Before running the proposed disjunctive optimization model, we again simulated the corridor in order to observe the impact of the demand increment over it. The corresponding contour plots are shown in Fig. 12. In this situation, an important increment in occupancy levels occurs, especially in the up direction. Concretely, the increment of demand implies high occupancy levels between stations 4 and 7 and services t 3 to t8 in the up direction. In the opposite direction the vehicle occupancy also increases, both in the spatial and time dimensions. The AWT increases up to 9.90 minutes in the up direction and to 8.33 minutes in the down direction. This corresponds to an increment of about $50 \%$ in AWT in both directions.

Finally, in order to obtain a new solution, we have solved the short-turning optimization model by incorporating short-turning services. Considering the new load profiles in both directions (see Fig. 11), the number of short-turning services between full-length ones required to capture the increment of demand is $N=1$. The model was programmed in GAMS and was solved by a branch-and-cut algorithm using AlphaECP (Extended Cutting Planes Westerlund and Pörn 2002) on a computer with an Intel i3 processor and 4 GB of RAM. Computational time was about 25 seconds. The optimal solution proposes turn-back points at stations 3 and 7 and an offset of two minutes for short-turning services. This solution gives rise to the inclusion of two short-turning services in the up direction and of two such services in the opposite direction. These are located before services 5 and 6 . In order to analyze the impact of the short-turning services over the corridor, we have simulated the new obtained solution. The results are shown in Fig. 13.

As illustrated in this figure, occupancy levels revert to a situation similar to the initial one. The impact of the increment demand matrix is absorbed by the short-turning services and the AWT decreases to 6.94 and 5.21 minutes for the up and down directions, respectively.

We compare the results of inserting train units performing short-turning services with respect to the insertion of the same number of train units performing full-length services. Figure 14 shows the occupancy levels obtained by using full-length services instead of shortturn ones. By the inclusion of full-length services we obtain an AWT of 8.73 minutes in the up direction and of 7.16 in the down direction (see Table 1). Since we have considered a demand increment $O^{\prime}$ which is not concentrated in just one area, occupancy levels are slightly better with full-length services because they capture all the demand outside the short-turn zone. However, this difference is insignificant in comparison with the differences between 

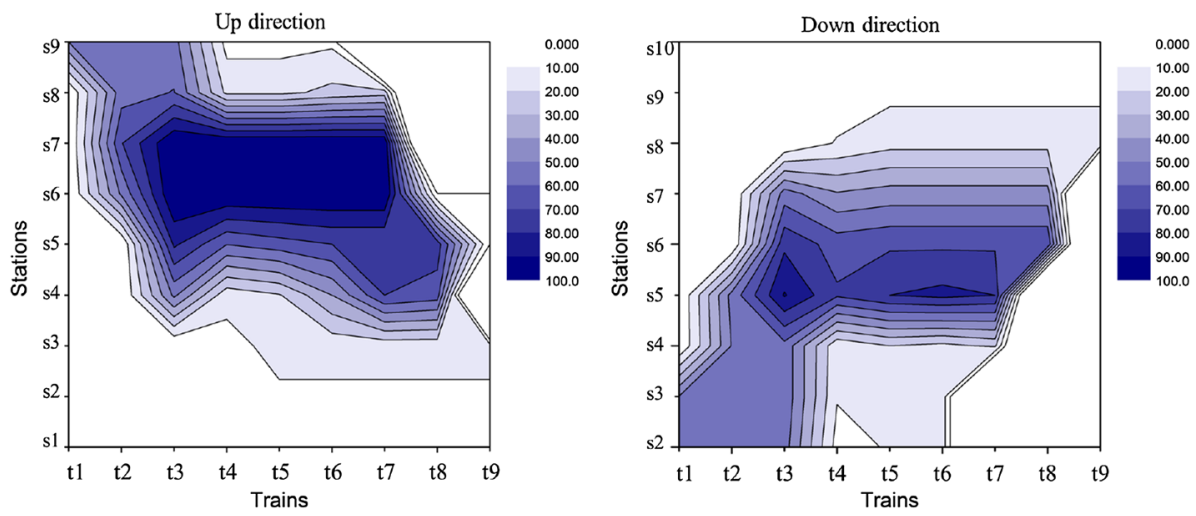

Fig. 13 Occupancy map corresponding to the timetable with short-turning services and demand matrix $O+O^{\prime}$
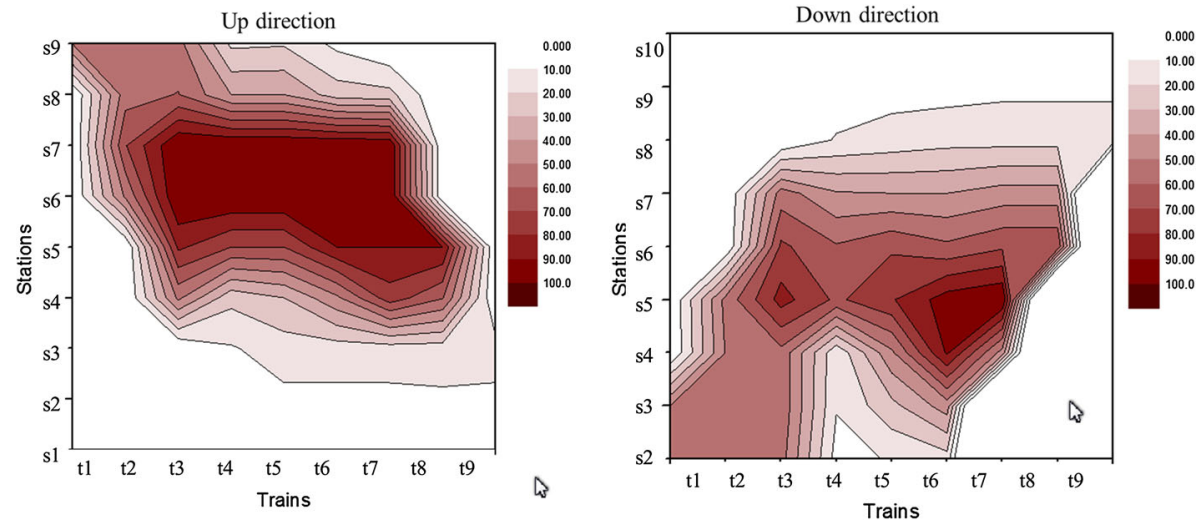

Fig. 14 Occupancy map considering full-length services and demand matrix $O+O^{\prime}$

Table 1 AWT after the demand increment, i.e., with demand matrix $O+O^{\prime}$

\begin{tabular}{lll}
\hline & Direction & AWT \\
\hline Initial timetable & Up & 9.9 \\
& Down & 8.33 \\
Initial timetable + short-turning & Up & 6.94 \\
& Down & 5.21 \\
Initial timetable + full-length & Up & 8.73 \\
& Down & 7.16 \\
\hline
\end{tabular}

the AWT obtained with the two policies (see Tables 1 and 2), which are clearly smaller when considering the short-turning policy. These results are consistent with the proposed model structure since the selection of a set of full-length services is a particular case of short-turning services where turn-back stations are the first and the last stations of the line. 
Table 2 Improvement of the AWT when considering short turning policy

\begin{tabular}{lllc}
\hline & Direction & Initial timetable & $\begin{array}{c}\text { Initial timetable }+ \\
\text { full-length }\end{array}$ \\
\hline $\begin{array}{l}\text { Initial timetable }+ \\
\text { short-turning }\end{array}$ & Up & $29.90 \%$ & $20.50 \%$ \\
& Down & $37.45 \%$ & $27.23 \%$ \\
\hline
\end{tabular}

\section{Conclusions}

We have considered the problem of inserting special short-turning services in a two-way railway line in order to make an efficient use of the available trains. To this end, we have developed an optimization model which integrates short-turning shuttle operations, thus yielding higher frequencies on certain critical segments, while maintaining the timetable of previously programmed services. Turn-back points location, departure and arrival times and short-turning offsets were obtained by solving a mixed integer linear optimization model. Experiments conducted on a real line of the Madrid commuter railway system show the benefit of short-turning policies in mitigating the increase in AWT resulting from an increased demand. We have shown that by adding only one short service between two long ones (during the disturbance interval) with an adequate location of the turn-back points and offsets, the AWT is reduced by $29.9 \%$ for the up direction and by $37.45 \%$ for the down direction. When comparing a short turning policy against a full-length one, we obtain improvements of $20.5 \%$ in the up direction and of $27.23 \%$ in the down direction. This approach can be useful at the tactical and operational levels since disruptions based on demand increments may either be known in advance or unexpected. Neverteless, sufficient advance warning is necessary to transport the reserve units to the short-turning zone. If the event is expected, this can be done easily in advance, but in the case of unexpected events, the implementation of the solution is more difficult and it may even be useless if the event has a short duration. In this work, we have considered a single short-turning zone. More short-turning zones could be simultaneously selected (Eq. (4)) by distinguishing between interacting and non-interacting cases, as well as taking into consideration whether the number of zones is known or unknown. In the last case, the number of zones would be a variable, which would add complexity to the model. As a byproduct of this work we have provided a simulation tool to estimate the trains occupancy levels which takes into account train capacities, as well as passengers boarding and alighting the trains.

Acknowledgments This research work was partially supported by the Excellence program of the Andalusian Government, under grant P09-TEP-5022, and by the Natural Sciences and Engineering Research Council of Canada (NSERC) under grant 39682-10. We are also grateful to RENFE (Madrid commuter railway system operator) for providing data and information relevant to the calibration of the model. Thanks are due to the referees for their valuable comments.

\section{References}

Abril, M., Barber, F., Ingolotti, L., Lova, A., Salido, M. A., \& Tormos, P. (2004). An efficient method to schedule new trains on a heavily loaded railway network. In C. Lemaîitre, C. A. Reyes, \& J. A. González (Eds.), Advances in Artificial Intelligence-IBERAMIA 2004 (Vol. 3315, pp. 164-173)., Lecture Notes in Computer Science Berlin Heidelberg: Springer.

Burdett, R. L., \& Kozan, E. (2009). Techniques for inserting additional trains into existing timetables. Transportation Research Part B: Methodological, 43(8-9), 821-836. 
Bussieck, M. R. (1998). Optimal lines in public rail transport. PhD thesis, Technische Universität Braunschweig, Germany.

Bussieck, M. R., Winter, T., \& Zimmermann, U. T. (1997). Discrete optimization in public rail transport. Mathematical Programming, 79(1-3), 415-444.

Cacchiani, V., Caprara, A., \& Toth, P. (2010a). Non-cyclic train timetabling and comparability graphs. Operations Research Letters, 38(3), 179-184.

Cacchiani, V., Caprara, A., \& Toth, P. (2010b). Scheduling extra freight trains on railway networks. Transportation Research Part B: Methodological, 44(2), 215-231.

Cadarso, L., \& Marín, A. (2012). Integration of timetable planning and rolling stock in rapid transit networks. Annals of Operations Research, 199(1), 113-135.

Canca, D. (2009). Operación mixta de una línea de ferrocarril. Análisis de capacidad y gestión de material rodante. Dirección y Organización, 39, 45-53.

Canca, D., Barrena, E., Algaba, E., \& Zarzo, A. (2014). Design and analysis of demand-adapted railway timetables. Journal of Advanced Transportation, 48(2), 119-137.

Canca, D., Barrena, E., Zarzo, A., Ortega, F. A., \& Algaba, E. (2012). Optimal train reallocation strategies under service disruptions. Procedia Social and Behavioral Sciences, 54, 402-413.

Ceder, A. (1989). Optimal design of transit short-turn trips. Transportation Research Record, 1221, 8-22.

Ceder, A., \& Wilson, N. H. M. (1986). Bus network design. Transportation Research Part B: Methodological, 20(4), 331-334.

Chen, M., Liu, X., \& Xia, J. (2005). Dynamic prediction method with schedule recovery impact for bus arrival time. Transportation Research Record, 1923, 208-217.

Chen, M., Niu, H. (2009). Modeling transit scheduling problem with short-turn strategy for a congested public bus line. In R. Liu, J. Zhang, and C. Guan, editors, Logistics: The Emerging Frontiers of Transportation and Development in China. Eighth International Conference of Chinese Logistics and Transportation Professionals (ICCLTP), pp. 4320-4326. American Society of Civil Engineers.

Chierici, A., Cordone, R., \& Maja, R. (2004). The demand-dependent optimization of regular train timetables. Electronic Notes in Discrete Mathematics, 17, 99-104.

Coor, G. T. (1997). Analysis of the Short-Turning Strategy on High-Frequency Transit Lines. PhD thesis, Masschusetts Institute of Technology.

Corman, F., D’Ariano, A., Pacciarelli, D., \& Pranzo, M. (2012). Bi-objective conflict detection and resolution in railway traffic management. Transportation Research Part C: Emerging Technologies, 20, 79-94.

Cortés, C. E., Jara-Díaz, S., \& Tirachini, A. (2011). Integrating short turning and deadheading in the optimization of transit services. Transportation Research Part A: Policy and Practice, 45(5), 419-434.

D’Ariano, A., Pacciarelli, D., \& Pranzo, M. (2007). A branch and bound algorithm for scheduling trains in a railway network. European Journal of Operational Research, 183(2), 643-657.

Delle Site, P. D., \& Filippi, F. (1998). Service optimization for bus corridors with short-turn strategies and variable vehicle size. Transportation Research Part A: Policy and Practice, 32(1), 19-28.

Desaulniers, G., Hickman, M. (2007). Public transit. In C. Barnhart and G. Laporte, editors, Transportation, volume 14 of Handbooks in Operations Research and Management Science, chapter 2, pp. 69-127. Amsterdam: Elsevier.

Eberlein, X. J., Wilson, N. H. M., \& Bernstein, D. (1999). Modeling real-time control strategies in public transport operations. In N. H. M. Wilson (Ed.), Computer-aided Transit Scheduling (Vol. 471, pp. 352346), Lecture Notes in Economics and Mathematical Systems Springer: Berlin Heidelberg.

Eberlein, X. J., Wilson, N. H. M., Barnhart, C., \& Bernstein, D. (1998). The real-time deadheading problem in transit operations control. Transportation Research Part B: Methodological, 32(2), 77-100.

Fischetti, M., Salvagnin, D., \& Zanette, A. (2009). Fast approaches to improve the robustness of a railway timetable. Transportation Science, 43(3), 321-335.

Flier, H., Graffagnino, T., \& Nunkesser, M. (2009). Scheduling additional trains on dense corridors. In J. Vahrenhold (Ed.), Experimental Algorithms (Vol. 5526, pp. 149-160)., Lecture Notes in Computer Science Springer: Berlin Heidelberg.

Fu, L., Liu, Q., \& Calamai, P. (2003). Real-time optimization model for dynamic scheduling of transit operations. Transportation Research Record, 1857, 48-55.

Furth, P. G. (1987). Short turning on transit routes. Transportation Research Record, 1108, 42-52.

Grosfeld-Nir, A., \& Bookbinder, J. H. (1995). The planning of headways in urban public transit. Annals of Operations Research, 60, 145-160.

Guihaire, V., \& Hao, J. K. (2008). Transit network design and scheduling: A global review. Transportation Research Part A: Policy and Practice, 42(10), 1251-1273.

Hadas, Y., \& Ceder, A. (2010). Optimal coordination of public-transit vehicles using operational tactics examined by simulation. Transportation Research Part C: Emerging Technologies, 18(6), 879-895. 
Kroon, L. G., Dekker, R., \& Vromans, M. (2007). Cyclic railway timetabling: a stochastic optimization approach. In F. Geraets, L. Kroon, A. Schöbel, D. Wagner, \& C. D. Zaroliagis (Eds.), Algorithmic Methods for Railway Optimization (Vol. 4359, pp. 41-66)., Lecture Notes in Computer Science Berlin Heidelberg: Springer.

Liebchen, C. (2004). Symmetry for periodic railway timetables. Electronic Notes in Theoretical Computer Science, 92, 34-51.

Liebchen, C., \& Möhring, R. (2002). A case study in periodic timetabling. Electronic Notes in Theoretical Computer Science, 66(6), 1-14.

Liebchen, C., \& Möhring, R. (2007). The modeling power of the periodic event scheduling problem: railway timetables and beyond. In F. Geraets, L. G. Kroon, A. Schöbel, D. Wagner, \& C. D. Zaroliagis (Eds.), Algorithmic Methods for Railway Optimization (Vol. 4359, pp. 3-40)., Lecture Notes in Computer Science Springer: Berlin Heidelberg.

Liebchen, C., \& Peeters, L. (2009). Integral cycle bases for cyclic timetabling. Discrete Optimization, 6(1), 89-109.

Liebchen, C., \& Stiller, S. (2009). Delay resistant timetabling. Public. Transport, 1(1), 55-72.

Louwerse, I., \& Huisman, D. (2014). Adjusting a railway timetable in case of partial or complete blockades. European Journal of Operational Research, 235(3), 583-593.

Magnanti, T. L., \& Wong, R. T. (1984). Network design and transportation planning: Models and algorithms. Transportation Science, 18(1), 1-55.

Mannino, C., \& Mascis, A. (2009). Optimal real-time traffic control in metro stations. Operations Research, 57(4), 1026-1039.

Mesa, J. A., Ortega, F. A., \& Pozo, M. A. (2009). Effective allocation of fleet frequencies by reducing intermediate stops and short turning in transit systems. In R. K. Ravindra K. Ahuja, R. H. Möhring, and C. D. Zaroliagis, (Ed.), Robust and Online Large-Scale Optimization (Vol. 5868, pp. 293-309)., Lecture Notes in Computer Science Springer: Berlin Heidelberg.

Mesa, J. A., Ortega, F. A., \& Pozo, M. A. (2013). Locating optimal timetables and vehicle schedules in a transit line. Annals of Operations Research, 144(1), 263-285. doi:10.1007/s10479-013-1393-5 (published on line 25 .

Michaelis, M., \& Schöbel, A. (2009). Integrating line planning, timetabling, and vehicle scheduling: A customer-oriented heuristic. Public Transport, 1(3), 211-232.

Quak, C. B. (2003). Bus line planning. Master's thesis, Delft University of technology, The Netherlands.

Serafini, P., \& Ukovich, W. (1989). A mathematical model for periodic scheduling problems. SIAM Journal on Discrete Mathematics, 2(4), 550-581.

Soeldner, D.W. (1993). A Comparison of Control Options on the MBTA Green Line. PhD thesis, Massachusetts Institute of Technology.

Tirachini, A., Cortés, C.E. (2007). Disaggregated modeling of pre-planned short-turning strategies in transit corridors. In Transportation Research Board (TRB) 86th Annual Meeting, Washington DC., 21-25 Jan 2007.

Tirachini, A., Cortés, C. E., \& Jara-Díaz, S. R. (2011). Optimal design and benefits of a short turning strategy for a bus corridor. Transportation, 38(1), 169-189.

Vera, V.A. (2010). Integrating Crew Scheduling and Rostering Problems. PhD thesis, Alma Mater Studiorum Università di Bologna, Italy.

Vijayaraghavan, T. A. S., \& Anantharamaiah, K. (1995). Fleet assignment strategies in urban transportation using express and partial services. Transportation Research Part A: Policy and Practice, 29(2), 157-171.

Walker, C. G., Snowdonb, J. N., \& Ryana, D. M. (2005). Simultaneous disruption recovery of a train timetable and crew roster in real time. Computers \& Operations Research, 32(8), 2077-2094.

Wardman, M., Shires, J., Lythgoe, W., \& Tyler, J. (2004). Consumer benefits and demand impacts of regular train timetables. International Journal of Transport Management, 2(1), 39-49.

Westerlund, T., \& Pörn, R. (2002). Solving pseudo-convex mixed integer optimization problems by cutting plane techniques. Optimization and Engineering, 3(3), 253-280.

Wilson, N. H. M., Macchi, R. A., Fellows, R. E., \& Deckoff, A. A. (1992). Improving service on the MBTA Green Line through better operations control. Transportation Research Record, 1361, 296-304.

Zhou, X., \& Zhong, M. (2005). Bicriteria train scheduling for high-speed passenger railroad planning applications. European Journal of Operational Research, 167(3), 752-771. 